\title{
Anaemia due to gastric haemorrhage by a metastatic Merkel cell carcinoma
}

\author{
Jorge Santos-Juanes ${ }^{1}$, Carmen Palomo², Luis Rodrigo³ ${ }^{3}$ Iván Fernández-Vega ${ }^{4}$ \\ ${ }^{1}$ Pathology Department, Dermatopathology Section, Hospital Universitario Central de Asturias, Oviedo, Spain \\ IInternal Medicine Department, Hospital Universitario Central de Asturias, Oviedo, Spain \\ ${ }^{3}$ Gastroenterology Service, Hospital Universitario Central de Asturias, Oviedo, Spain \\ ${ }^{4}$ Hospital Universitario de Araba, Araba, Spain
}

Prz Gastroenterol 2015; 10 (4): 244-246

DOI: 10.5114/pg.2015.51183

\begin{abstract}
Address for correspondence: Iván Fernández-Vega MD, PhD, Hospital Universitario de Araba, Jacinto Arregui, 01010 Alava Araba, Spain, phone: +34 696527994, e-mail: ivan_fernandez_vega@hotmail.com
\end{abstract}

Merkel cell carcinoma (MCC) is an aggressive uncommon neuroendocrine tumour of the skin, probably originating from Merkel cells, and described by Toker in 1972 as Trabecular carcinoma [1]. Subsequently, electron-dense neurosecretory granules were demonstrated in the tumour cells and it was classified as definite neuroendocrine tumour. Merkel cell carcinoma is characterised by a high incidence of local recurrences, regional local metastases, distant metastasis, and high mortality rate [2]. Merkel cell carcinoma occurs predominantly in elderly white males (61\% male vs. $39 \%$ female), with a mean age of 69 years at diagnosis [3]. Lately, reports on MCC have increased, probable due to the higher incidence of damaging sun exposure, the advancing age of the population, and the increasing numbers of immunocompromised individuals [3]. In fact, it has been described that MCC is strongly associated with a polyomavirus [4], which might explain why MCC appears more frequently in immunocompromised patients such as solid-organ transplant recipients and HIV/AIDS infected people, and is associated with other malignancies [5]. Taking all of the data together, MCC could sometimes appear as a metastatic tumour in many different organs such as liver, bone, lung, and brain [6]. In light of this we report herein a patient who developed anaemia due to gastric haemorrhage as the first sign of gastric metastasis by MCC, which has rarely been reported [7].

A 52-year-old man with a previous diagnosis of type 2 diabetes mellitus and mild retinopathy was referred to our hospital due to severe asthaenia and dizziness. Almost 2 years previously, a cutaneous surgical excision was performed removing a $2 \mathrm{~cm}$ well-delimitated solid nodule on his right thigh, diagnosed as a Merkel cell carcinoma after a battery of immunohistochemical tests (Figures 1 A, B). Moreover, ipsilateral inguinal lymphadenectomy was done revealing one infiltrated lymph node. The patient underwent local radiotherapy (5000 RADs) in combination with chemotherapy (carboplatin and etopoxide) with a very good clinical tolerance. So far, the patient has been asymptomatic.

The current physical examination of the patient pointed out a paleness of the skin and mucous membranes. An extensive workup was performed showing intense anaemia with haemoglobin level of $8.4 \mathrm{~g} / \mathrm{dl}$. Abdominal computed tomography (CT) highlighted a gastric wall thickening with associated regional lymphadenopathy. Subsequently, gastroscopy showed a bleeding, ulcerated lesion measuring $2 \mathrm{~cm}$ in diameter, with irregular borders and presented at minor curvature of the stomach. Biopsies were taken from the lesion and studied in the Pathology Department.

Microscopic examination of the gastric ulcer showed an gastric mucosa infiltrated by small cells with uniform hyperchromatic nuclei with "salt and pepper" chromatin (Figures 1 C, D). Immunohistochemically, these cells were positive for cytokeratin 8, cytokeratin 20 (dot-like pattern), neuron-specific enolase, synaptophysin, and chromogranin A (Figures 1 E, F). Ki-67 proliferation index was about $50 \%$. The tumour cells were negative for CK7, TTF1, S100, CD45, and CD99. Moreover, a previous surgical skin biopsy diagnosed as MCC almost 2 years ago was fully revised, pointing out the same histological appearances and immunohistochemistry expressions.

Finally, these clinical, radiological, and pathological findings were consistent with an ulcerative metastatic MCC in the stomach. The patient was transfused with two red blood cell concentrates and oral iron. Even though he received chemotherapy again, he passed away 6 months after admission.

Merkel cell carcinoma is an rare neuroendocrine tumour of the skin that occurs predominantly in the head 

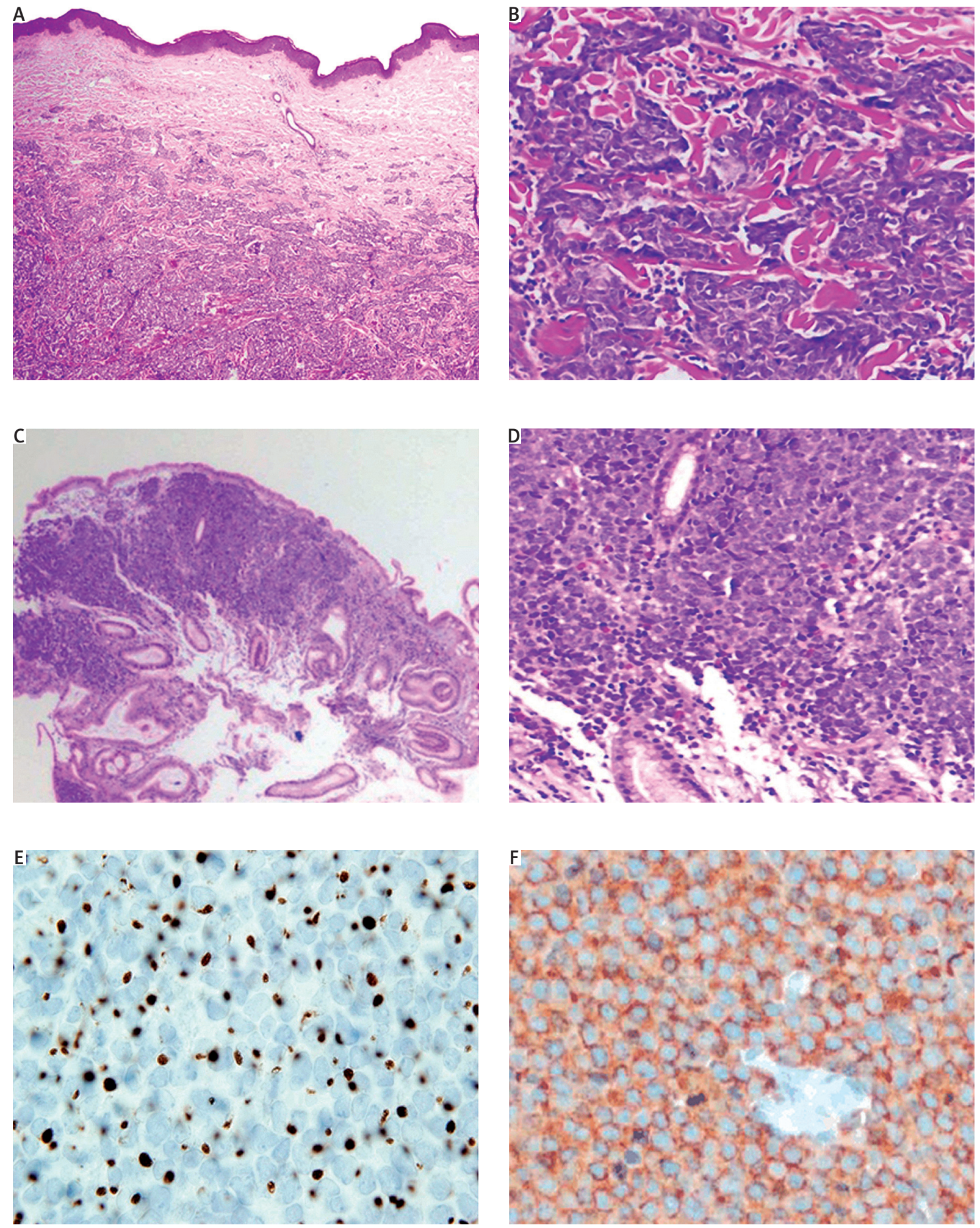

Figure 1. Histopathological images of the primary and metastatic Merkel cell carcinoma. Haematoxylin and eosin staining of the primary cutaneous MCC (A, 40x; B, 200x) and the gastric metastasize (C, 20x; D, 200x) show an infiltrative small round cell tumour. Tumour cells show a cytoplasmic dot-like staining pattern for cytokeratin 20 (E, 400x) and chromogranin A (F, 400x). All immunostaining was performed by immunoperoxidase technique on paraffin-embedded tissue sections 
and neck (41\% to 50\%), followed by the extremities (32\% to $38 \%$ ) and trunk (12\% to $14 \%$ ), mainly in elderly white males [3]. This neoplasm usually presents as a rapidly growing, dome-shaped red or bluish nodule. However, sometimes it can adopt a plaque-like appearance with small satellite lesions. Thus, due to its rareness and heterogeneous clinical appearance, it is hardly ever thought of as a primary diagnosis, so definite diagnosis is often established only after biopsy [8]. Therefore, on $\mathrm{H}+\mathrm{E}$, microscopic appearances are consistent with highly proliferative cells of uniform small round cell with atypical features that could be easily confused with lymphoma, leukaemia, metastatic small cell carcinoma of the lung, melanoma, and other poorly differentiated cutaneous malignancies [9]. However, MCC presents a few microscopic clues that could help us to make an accurate diagnosis, such as pseudorosettes or a rounded irregular nucleus with condensed and uniformly dispersed nuclear chromatin, without the presence of any nucleoli. Immunohistochemically, the tumour cells are mainly characterised by their positive expression for neuroendocrine markers such as synaptophysin and chromogranin, with a characteristic diagnostic feature of a cytoplasmic dot-like staining pattern for cytokeratin 20 [10]. Moreover, negativity for CK7, TTF1, S100, CD45, and CD99 excluded other differential diagnosis, such as metastatic small cell carcinoma of the lung, melanoma, lymphoma, or primitive neuro-ectodermal tumours, respectively. None of the plasma hormone levels was elevated, considering the remote possibility of a primary neuroendocrine tumour of the stomach.

Merkel cell carcinoma is an aggressive tumour characterised by high incidence of local recurrences of regional local metastases and distant metastasis. In fact, the most common sites of distant metastasis are distant lymph nodes (27-60\%), distant skin (9-30\%), lung (10$23 \%)$, central nervous system (18\%), and bone (15\%) [11]. Merkel cell carcinoma rarely metastasises to the stomach. However, a few cases have been reported in the literature with an interesting recent study performed by Syal et al., pointing out that up to $80 \%$ of patients with gastric metastasis of MCC presented with upper gastrointestinal bleeding consistent with the presented case [12]. Furthermore, they also noted that the mean time between upper gastrointestinal bleeding due to a metastatic MCC and death is about 4 months [12], slightly less than the 6-month survival observed in our patient.

In conclusion, MCC is an aggressive skin tumour that hardly ever metastasises to the stomach, giving rise to a fatal outcome. Immunohistochemical studies play an important role in the diagnosis, in order to rule out more frequent gastric malignancies. An interdisciplinary approach is recommended in the management of this rare tumour.

\section{Conflict of interest}

The authors declare no conflict of interest.

\section{References}

1. Tang CK, Toker C, Nedwich A, et al. Unusual cutaneous carcinoma with features of small cell (oat cell-like) and squamous cell carcinomas. A variant of malignant Merkel cell neoplasm. Am J Dermatopathol 1982; 4: 537-48.

2. Agelli M, Clegg LX, Becker JC, et al. The etiology and epidemiology of merkel cell carcinoma. Curr Probl Cancer 2010; 34: 14-37.

3. Duprat JP, Landman G, Salvajoli JV, et al. A review of the epidemiology and treatment of Merkel cell carcinoma. Clinics (Sao Paulo) 2011; 66: 1817-23.

4. Feng H, Shuda M, Chang Y, et al. Clonal integration of a polyomavirus in human Merkel cell carcinoma. Science 2008; 319: 1096-100.

5. Kaae J, Hansen AV, Biggar RJ, et al. Merkel cell carcinoma: incidence, mortality, and risk of other cancers. J Natl Cancer Inst 2010; 102: 793-801.

6. Raaf JH, Urmacher C, Knapper WK, et al. Trabecular (Merkel cell) carcinoma of the skin. Treatment of primary, recurrent, and metastatic disease. Cancer 1986; 57: 178-82.

7. Parikh MP, Samo S, Ganipisetti V, et al. Gastric metastasis of Merkel cell carcinoma, a rare cause of gastrointestinal bleeding: case report and review of the literature. J Gastrointest Oncol 2014; 5: E68-72.

8. Lemos BD, Storer BE, lyer JG, et al. Pathologic nodal evaluation improves prognostic accuracy in Merkel cell carcinoma: analysis of 5823 cases as the basis of the first consensus staging system. J Am Acad Dermatol 2010; 63: 751-61.

9. Idowu MO, Contos M, Gill S, et al. Merkel cell carcinoma: a report of gastrointestinal metastasis and review of the literature. Arch Pathol Lab Med 2003; 127: 367-9.

10. Li M, Liu C. Cytokeratin 20 confirms merkel cell metastasis to stomach. Appl Immunohistochem Mol Morphol 2004; 12: 346-9.

11. Medina-Franco H, Urist MM, Fiveash J, et al. Multimodality treatment of Merkel cell carcinoma: case series and literature review of 1024 cases. Ann Surg Oncol 2001; 8: 204-8.

12. Syal NG, Dang S, Rose J, et al. Gastric metastasis of merkel cell cancer: uncommon complication of a rare neoplasm. J Ark Med Soc 2012; 109: 134-6.

Received: 8.09 .2014

Accepted: 29.12 .2014 